\title{
Variants of Blood Supply Sources of Kidney Segments at Dychomatic Division of the Renal Artery
}

\author{
Vagabov I. U.* Vezirkhanov A.Z. Kafarov E.S. \\ Department of normal and topographic anatomy with operational surgery, Chechen State University, Grozny, Russia \\ *Corresponding author. Email: malsi_85@mail.ru
}

\begin{abstract}
The aim of the study was to carry out a three-dimensional quantitative analysis of blood supply variants of kidney segments during dichotomous division of the renal artery. The study prepared 116 corrosive preparations of human kidney arterial system, followed by 3D scanning to obtain digital models. It identified types of branching of the main branches of the renal artery in three-dimensional projection, a number of vessels of the branches of the renal artery of different orders, depending on the types of branching of each main branch. The study determined segmental arteries, their number involved in the nutrition of the renal segments, depending on the types of intraorgan branching: in the main and loose types of branching. The morphometric analysis data were processed by the methods of variation statistics. It was found that in a kidney, regardless of the type of two-zone blood supply, in the nutrition of pole segments, functions 1 segmental artery $(50.1 \%), 2$ arteries $(35.3 \%), 1$ artery originating from the renal artery $(7.5 \%)$ and 1 artery from the aorta $(6.2 \%)$. In the loose type of branching of pole arteries, pole segments have 2 segmental arteries more often than in the main one. On average, with this type of branching, they are more often involved in feeding pole segments than in the trunk type $(76.5 \%$ and $23.5 \%)$, respectively. In the upper anterior and lower anterior segments, they branch on average: 1 segmental artery (91.1 \%) or 2 arteries $(8.8 \%)$. In the posterior segment, on average, they branch: 1 artery $(86.7 \%), 2$ arteries $(12.2 \%), 3$ arteries $(1.6 \%)$, which is important to take into account when performing surgical interventions on the kidneys.
\end{abstract}

Keywords: kidney, angioarchitectonics, segments

\section{INTRODUCTION}

Many researchers, both domestic and foreign, have studied kidney zonal and segmental structure, as well as its blood supply [1-7, 9-17]. According to many researchers, the determining factor in the division of all kidneys into segments is its arterial bed, namely the arterial system of the kidney, which gives rise to segmental arteries [1-7, 9-17].

The variability of the blood supply to the renal segments was studied by Ajmani (1983) (cited by A.N. Ponukalin et al., 2013). According to the author's data, the main renal artery at the renal hilus is divided into ventral and dorsal arteries in 98 $\%$ of cases, and this division enters the renal hilus before the renal artery. The author identified 5 variants of division of the ventral and 3 variants of division of the dorsal branch of the main renal artery. The author also found that the upper pole segment of the kidney could have several variants of blood supply (about 7 variants) $[9,12]$. According to some data, the number of segments can reach up to six (Olofinsky, L.A., Sabirov, S.R. and others) (cited by Ponukalin, A. N. et al., 2013) [9]. According to this author, kidneys can have 4 segments (3.5\% of cases); in $72.6 \%$ of cases it can have 5 segments and in $23.9 \%$ of cases in kidneys there can be up to 6 segments. Sabirov, S.R. (1978) identified 4 segments in $35 \%$ of cases, 5 segments of the kidney in $38.5 \%$ of cases, and 6 segments in $26.5 \%$ of cases [10].
Of course, both domestic and foreign scientists have dealt with the issues of the segmental structure of a kidney. According to Longia (1982) (cited by Ponukalin, A. N. et al., 2013), a kidney has five segments in $53 \%$ of cases $[9,10]$. In $46 \%$ of cases, a kidney has a four-segment structure and $1 \%$ of cases has a three-segment structure. The author identified variants of kidneys with the presence of less than five segments. According to the author, in $15 \%$ of observations there is no upper pole segment; in $14 \%$ of cases, the anterior lohase segment was a combined superior and inferior anterior segments; in the third variant, the lower pole segment is absent, which was found in $17 \%$ of cases $[9,10]$.

Sampaio $(1993,1996)$ also studied variants of the segmental structure of the kidneys (cited by Ponukalin, A.N. et al., 2013) [9, 16]. According to this author, in $61.2 \%$ of cases, a kidney has 5 segments, and 4 segments in $38.8 \%$ of cases. In $73.5 \%$ of cases, kidneys have an upper segment, which occupied an area of $13 \%$. In $61.2 \%$ of cases, kidneys have an upper and lower anterior lobe segments, with an area of $21.4 \%$ and $17.2 \%$, respectively. In $38.8 \%$ of cases, kidneys have one anterior lobe segment and occupy an area of $28.4 \%$. Some kidneys have the inferior pole and retilochanteric segments, which occupied an area of $22.2 \%$ and $33.8 \%$, respectively $[9,16]$. 
Thus, there are various data on the variants of the segmental structure of kidneys. In general, there are different variants of blood supply to its segments, which require their careful analysis. The questions of sources and number of segmental arteries in different variants of renal artery division have not yet been clarified.

\section{METHODS AND MATERIALS}

116 corrosive preparations of human renal arterial vessels were prepared for the study. The corrosive specimens were 3D scanned to produce digital models.

1) The computer program "Mimics-8.1" identified:

- projection of the main arteries in the hilum of the kidneys in relation to the planes;

- $\quad$ projection of extraorgan branches of the renal artery;

- number of main branches of the renal arteries at the hilum of the kidney;

2) It also determined:

- types of intraorgan branching of the main branches of the renal artery inside the kidney, depending on the division options at the gate of each of its branches:

- with the main type:

- $\quad$ with loose type;

3) It also determined:

- the number of vessels of the main branches of the renal artery of different orders:

- $\quad$ with the main type;

- $\quad$ with loose type;

4) The study identified segmental arteries, their number involved in the nutrition of the renal segments, depending on the types of intraorgan branching:

- with the main and loose types of branching.

Statistical processing included the calculation of the main indicators of random variables distribution: median, mean, quartiles, confidence interval, minimum and maximum values, variance, standard deviation, mean error, median error. We used a licensed package of applied statistical programs MedStat in accordance with the recommendations [8].

\section{RESULTS}

It was found that in $54.2 \%$ of cases, a variant of dichotomous division of the main renal artery into ventral and dorsal branches is observed more often.
The authors conducted three-dimensional (3D) and stereoanatomical analysis of links of the arterial bed of kidneys, taking into account the types of branching of intraorgan vessels. It showed that with the first type of branching of intraorgan arterial vessels, the ventral branch of the renal artery was divided according to the loose type, and the dorsal branch along the main one, which was revealed in $46.2 \%$ of cases. At the same time, the level organization of the renal artery system' links with this variant and the type of branching of the arterial vessels of the kidney showed that the number of segmental arteries "A. interlobares -1 " (3) averaged $-(X \pm m) 7 \pm 1$.

Five-segmental structure of kidneys with this variant and the type of branching of its intraorgan arteries, the upper polar segment was supplied with blood in the first variant by one segmental artery - "A. interlobares - 1" (3), extending from the ventral branch - "A. ventralis" (zonal) (2), (41.2\% of cases). This artery was mostly distributed in the ventral half of the upper pole of the kidney and in the smaller part of the dorsal. In the second variant, this segment was supplied with blood by two segmental arteries extending from the ventral and dorsal branches (32.3\% of cases), distributed by their branches in the ventral and dorsal parts of the upper pole of kidneys. In the third variant, one segmental artery was distributed in the upper pole segment, extending from the main renal artery - "A. renalis" (1), $(21.2 \%)$ and in the fourth variant, this segment was supplied with blood by one segmental artery extending from the dorsal branch - "A. dorsalis" (zonal) (2), which was detected in $5.3 \%$ of cases. Moreover, its branches were mostly distributed in the dorsal half of the upper pole segment.

With this variant and type of branching of intraorganic arteries, kidneys were directed to the upper anterior and lower anterior segments along one segmental artery from the ventral branch of the main renal artery, evenly distributed by their branches in the upper and lower parts of the central part of the renal parenchyma. In the first variant, the lower pole segment was supplied with one segmental artery - "A. interlobares - 1" (3), extending from the ventral branch ( $68.3 \%$ of cases), distributed by its branches mostly in the ventral and smaller part of dorsal parts of the lower pole of kidneys. In the second variant, the blood supply to the lower pole segment involved two segmental arteries extending from the ventral and dorsal branches (27.4\% of cases), distributed in the ventral and dorsal parts of the lower pole of the kidney. In the third variant, this segment was fed by one segmental artery extending from the main renal artery - "A. renalis" (1), which was detected in $4.3 \%$ of cases.

The blood supply to the posterior segment was due to one segmental artery, which is a continuation of the dorsal branch - "A. dorsalis" (zonal) (2) of the main renal artery, being distributed by its branches in the parenchyma of the posterior segment.

In $23.8 \%$ of cases, the second type of branching of the intraorgan arterial system of kidneys was revealed, where both branches were divided according to the loose type, being distributed in the ventral and dorsal parts of the renal parenchyma. At the same time, the level organization of 
the links of the renal artery system with this variant and the type of branching of the arterial vessels of the kidneys showed that the number of segmental arteries "A. interlobares -1 " (3) averaged - (X \pm m) $9 \pm 1$.

Five-segmental structure of kidneys with this variant and the type of branching of its intraorgan arteries, the upper pole segment was supplied with blood in the first variant by two segmental arteries extending from the ventral branch - "A. ventralis" (zonal) (2), (36.2\% of cases). The branches for the most part were evenly distributed in the ventral and dorsal parts of the upper pole of the kidney. In the second variant, two segmental arteries were also distributed in this segment - "A. interlobares 1" (3), extending from the ventral and dorsal branches ( $31.4 \%$ of cases). In this case, the segmental arteries were evenly distributed both in the ventral and in the dorsal part of the upper pole of kidneys. In the third variant, the upper pole segment was fed by one segmental artery extending from the ventral branch - "A. ventralis" (zonal) (2), ( $24.3 \%$ of cases) or one segmental artery extending from the dorsal branch - "A. dorsalis" (zonal) (2), which was revealed in $8.1 \%$ of cases in the 4 th variant.

In the first variant, two segmental arteries extending from the ventral branch ( $66.5 \%$ of cases) or one segmental artery extending from the ventral branch were directed to the upper anterior segment - "A. ventralis" (zonal) (2), which was revealed in $33.5 \%$ of cases in the second variant. One segmental artery was directed to the lower anterior segment - "A. interlobares - 1" (3), extending from the ventral branch (64.5\% of cases); in $35.5 \%$ of cases of the second option two segmental arteries extended from the ventral branch.

In the first variant, the blood supply to the lower pole segment happened thanks to 2 segmental arteries extending from the ventral and dorsal branches $(54.2 \%$ of cases). In this case, vessels were evenly distributed in the ventral and dorsal parts of the parenchyma of the lower pole of the kidney.

In the second variant, the blood supply occurred by two segmental arteries extending from the ventral branch "A. ventralis" (zonal) (2), (37.3\% of cases) or due to one segmental artery - "A. interlobares - 1" (3), departing from the main renal artery - "A. renalis" (1), which was found in $8.5 \%$ of cases in the third variant. The posterior segment of the kidney was supplied with blood in the first variant by one segmental artery - "A. interlobares - 1" (3), extending from the dorsal branch - "A. dorsalis" (zonal) (2) $(58.5 \%$ of cases). In the second variant, two segmental arteries extended from the dorsal branch, which was met in $41.5 \%$ of cases.

In $19.4 \%$ of cases, the third type of arterial vessel branching was identified, where both branches of the renal artery system had a main branching type. At the same time, level organization of links of the renal artery system with this variant and the type of branching of the arterial vessels of the kidney showed that the number of segmental arteries "A. interlobares - 1" (3) averaged - $(\mathrm{X} \pm \mathrm{m}) 6 \pm 1$. In this case, the blood supply to the upper pole segment happened through one segmental artery - "A. interlobares - 1" (3), extending from the ventral branch (95.7\% of cases), which was revealed in the first variant. In the second variant, blood supply happened through one segmental artery extending from the main renal artery - "A. renalis" (1), which was found in $4.3 \%$ of cases. With this variant and type of branching, one segmental artery was directed to the upper anterior and lower anterior segments - "A. interlobares - 1" (3), from the ventral branch of the main renal artery. It was later found in the anterior - upper and lower parts of the central part of the kidney.

In the first variant, blood supply to the lower pole segment occurred due to one segmental artery extending from the ventral branch - "A. ventralis" (zonal) (2), (91.7\% of cases); in the second variant, one segmental artery was involved in the blood supply - "A. interlobares - 1" (3), departing from the main renal artery - "A. renalis" (1), which was found in $8.3 \%$ of cases.

The blood supply to the posterior segment happened throygh one segmental artery - "A. interlobares - 1" (3), which is, as a continuation of the dorsal branch of the main renal artery - "A. renalis" (1).

\section{CONCLUSION}

Thus, our three-dimensional (3D) stereoanatomical analysis showed that in a five-segment kidney in a two-zone (ventral and dorsal) blood supply system (i.e., a dichotomous division), the upper pole segment is supplied $(\mathrm{X} \pm \mathrm{m})$ by one segmental artery extending from the ventral branch $(46.2$ $\%)$. It can also be supplied by two segmental arteries extending from the ventral and dorsal branches (29.8\%); by one artery extending from the main renal artery $(10.5 \%)$ or by one segmental artery extending from the dorsal branch $(9.2 \%)$. The upper anterior segment is supplied with blood on the average $-(\mathrm{X} \pm \mathrm{m})$ by one segmental artery extending from the ventral branch $(83.3 \%)$ and by two segmental arteries extending from the ventral branch (16.6\%). The lower anterior segment is supplied with blood on the average $-(\mathrm{X} \pm \mathrm{m})$ due to one segmental artery extending from the ventral branch $(91.1 \%)$ and due to two segmental arteries extending from the ventral branch $(8.8 \%)$. The blood supply to the lower pole segment with this blood supply system occurred $(X \pm m)$ due to: one segmental artery extending from the ventral branch $(48.7 \%)$; $(\mathrm{X} \pm \mathrm{m})$ two segmental arteries extending from the ventral and dorsal branches $(28.5 \%)$; two segmental arteries extending from the ventral branch $(16.4 \%)$ or due to one artery extending from the main renal artery on average $-(\mathrm{X} \pm \mathrm{m})(8.3 \%)$. The posterior segment is supplied by one segmental artery, as a continuation of the dorsal branch $(86.7 \%)$ and by two arteries extending from the dorsal branch $(13.2 \%)$. The loose type of branching of the ventral branch, the main type of branching of the dorsal branch, and the segmental artery participates in the blood supply of the posterior single segment as a continuation of the dorsal artery. The dorsal artery immediately gives "subsegmental arteries" or arteries of the 4th order, which are important to take into account when performing surgical interventions on kidneys. 


\section{ACKNOWLEDGMENTS}

The article was published within the framework of the RFBR grant according to the agreement 19-315-90033.

\section{REFERENCES}

[1] M.P. Burykh, Anatomy of the calyx-pelvis complex of the human kidney in postnatal ontogenesis, vol. 84, Kharkov, 2000, p. 27.

[2] S.G. Eremeev, Arterial segments of the kidneys, Ph.D. Thesis, Voronezh, 1962, 17 p.

[3] O.K. Zenin, Human arterial system in numbers and formulas, Donbass, Donetsk, 2002, 196 p.

[4] O.K. Zenin, E.L. Kryukov, D.V. Remizov, Applied aspects of morphology. Actual aspects of functional morphology and integrative anthropology, Materials of Science and Practice. Conf., Vinnytsia, 20-21 May 2009, pp. 112-114.

[5] M.S. Kazartsev, Age features of the segmental structure of human kidneys, Ph.D. Thesis, Voronezh, 1969, 23 p.

[6] E.S. Kafarov, Variant anatomy of the renal artery and its branches, Ph.D. Thesis, Volgograd, 2004, 19 p.

[7] E.S. Kafarov, Structural transformations of the venous vessels of the human kidneys in mature, elderly and senile age, Ph.D. Thesis, Ufa, 2014, 45 p.

[8] Y.E. Lyakh, V.G. Guryanov, V.N. Khomenko et al., Fundamentals of computer biostatistics: analysis of information in biology, medicine and pharmacy using the statistical package MedStat, 2006, 214 p.

[9] A.N. Ponukalin, D.Y. Potapov, D.A. Durnov, Segmental structure and architectonics of the arterial bed of the kidney, Bull. of Med. Internet Conf. 3(4) (2013) 864-868. ISSN 2224-6150.

[10] S.R. Sabirov, Segmental structure of the human kidney, Ph.D. Thesis, Moscow, 1978, 18 p.

[11] V.V. Serov, Segmental structure of the vascular system of the kidney, Urology 3 (1959) 6-12.

[12] M.L. Ajmani, K. Ajmani, To study the intrarenal vascular segments of human kidney by corrosion cast technique, Anat. Anz. 154(4) (1983) 293-303.

[13] P.S. Bordei, D. Antohe, Anatomical study of triple renal arteries, Morpologie 86(274) (2002) 37-41.

[14] G.S. Longia, V. Kumar, S.K. Saxena, C.D. Gupta, Surface projection of arterial segments in the human kidney, Acta Anat (Basel) 113(2) (1982) 145-150.

[15] T. Pestemalci, A. Mavi, Y.Z. Yildiz et al., Bilateral triple renal arteries, Kidney Dis. Tranpl. 20(3) (2009) 468-470.

[16] F.J. Sampaio, J.L. Schiavani, L.A. Favorito, Proportional analysis of the kidney arterial segments, Urol. Res. 21(6) (1993) 371-374.

[17] D. Zahoi, V. Niculescu, Segmentarea renala concept morphologic cu valoare chirurgicala, The 4th National Congress of the Romanian Society of anatomists and the Congres of the Anatomy Departament of the Medical union of Balcans and Black Sea Countries, Oradea, Romania, 2000, 249 p.. 